\title{
Inequidad en el impuesto inmobiliario: análisis multicriterio (CRITIC) del valor catastral de vivienda en Mazatlán
}

\section{Inequity in real estate tax: multi-criteria analysis (CRITIC) of the cadastral value of housing in Mazatlan}

\author{
Luis Alfonso Colado Velázquez* \\ Josep Roca Cladera* \\ IVÁN HuMARÁn NAHED*
}

\begin{abstract}
TThe present document reports the results obtained from the spatial and CRITIC multi-criteria analysis performed to 1062 houses and the variables conforming the Cadastral Value of each one within the Cadastral Housing Categories ranging from M2 to M7 in Mazatlan, Sinaloa, Mexico. The weighting and the information for each criterion was obtained and used to analyze the current influence of each variable and how these allow to demonstrate the existence of inequity when obtaining the cadastral value of housing.
\end{abstract}

Keywords: land registry, cadastral value, inequity, CRITIC.

\section{Resumen}

En el presente documento se reportan los resultados obtenidos en el análisis multicriterio CRITIC y espacial realizado a 1062 viviendas en Mazatlán, Sinaloa (México); asimismo, se especifican las variables que conforman el Valor Catastral para cada una de las categorías catastrales de vivienda que van de M2 hasta M7, establecidas en el Instructivo de Valuación del estado de Sinaloa; donde se obtuvieron las ponderaciones de cada criterio y la información con la que se analiza la situación actual de influencia de cada variable y cómo, a partir de estos datos, se puede demostrar la inequidad al obtener el valor catastral de vivienda.

Palabras clave: catastro, valor catastral, inequidad, CRITIC.

\footnotetext{
*Universidad Autónoma de Sinaloa, correos-e: 1colado@hotmail.com e ivan.humaran@uas. edu.mx

** Universitat Politècnica de Catalunya, correo-e: josep.roca@upc.edu
} 


\section{Introducción}

Desde 1857, cuando se proyectaba la Constitución Política de los Estados Unidos Mexicanos, se postularon los principios de equidad y proporcionalidad, que quedaron plasmados en el documento final (Torres, 2006). Por su propia naturaleza jurídica, estos principios han sido estudiados con diferentes fines.

Para acercarnos al objetivo de este artículo, pretendemos dilucidar si existe una relación proporcional entre los valores catastrales y de mercado y, por otra parte, a partir de análisis previos sobre el tema, detectar si hay o no equidad en el cobro de los tributos inmobiliarios: Impuesto Predial, Impuesto sobre Adquisición de Inmuebles (ISAI) e Impuesto sobre la Renta (ISR), que necesitan del valor catastral. Cabe recordar que en México $y$ en el mundo el valor catastral es utilizado para fines fiscales, ya que es un valor de no mercado (IVSC, 2017). Asimismo, nuestro estudio se apoyará en técnicas matemáticas y estadísticas, que a la postre nos llevarán a conocer la afectación que se produce en los sectores sociales al momento de realizar la tributación inmobiliaria.

Es necesario aclarar que el trabajo no aborda un análisis de índole sociopolítico, ya que su finalidad primordial es contribuir con un estudio que demuestre las inconsistencias existentes en el valor catastral registrado en la Base de Datos (BD) del catastro en Sinaloa y como éste afecta en la tributación de manera inequitativa al contribuyente.

Así, el objetivo central de este documento es informar los resultados del análisis realizado al valor catastral en Mazatlán, mediante la ponderación de las variables del valor catastral y como éstas afectan al valor mismo, lo anterior mediante la aplicación de diferentes metodologías que nos permitan explicar de forma coherente y satisfactoria los resultados en el estudio, al detallar qué variables son las más influyentes en la estimación del valor catastral.

Metodológicamente se utilizó un análisis desde diversas perspectivas: se implementó la técnica Delphi, mediante la cual se realizó una consulta a los expertos en el área de valuación, cuyos resultados nos motivaron a interesarnos por esta problemática. Posteriormente, se obtuvo la base de datos (BD) con información de las categorías catastrales de vivienda, así como el valor calculado del mercado, tomando como base la información de la Asociación Mexicana de Profesionales Inmobiliarios (AMPI), que fue considerada como referencia máxima de valor; estos datos fueron tratados y analizados mediante ratios, que permitieron establecer el grado de coherencia entre el catastro y el mercado (AMPI).

Todas las muestras recopiladas en las categorías catastrales fueron analizadas mediante la metodología multicriterio, combinada con el 
método del ratio de valuación (IVSC, 2017); esto permite determinar tendencias que proporcionan información útil al valorar riesgos relativos a las operaciones comerciales dentro del contexto que se utilicen, así como conocer la ponderación de las variables predominantes en la obtención del valor catastral, obtenido con el Método CRITIC, (RATIO+CRITIC, CRITIC+RATIO) utilizado por Aznar et al. (2011).

Lo anterior posibilitó determinar el comportamiento objetivo de cada variable con respecto a la ponderación obtenida en cada categoría establecida en el estudio, evitando las dificultades que los encargados de generar los análisis económicos y financieros tienen para decidir con claridad la importancia relativa de los ratios obtenidos (Diakoulaki et al., 1995).

Con el resultado de esta metodología se observa qué variables han sido manipuladas para favorecer o desfavorecer algunas categorías y, de ese modo, constatar la inequidad con respecto a la estimación del valor catastral y, por consiguiente, la ilegalidad tributaria en el pago de los impuestos inmobiliarios.

\section{El problema de la no equidad y el catastro}

En este punto se cita la jurisprudencia constitucional administrativa P./J. 24/2000 de la Suprema Corte de Justicia de la Nación (SCJN) sobre "Impuestos. Principio de equidad tributaria previsto por el artículo 31, fracción IV, constitucional” (SJF, 2000), en la cual se menciona el pago de impuestos y determina en qué consiste el problema de inequidad; asimismo, esta tesis jurisprudencial aborda la obligación de crear categorías o clasificaciones de contribuyentes que no sean caprichosas, arbitrarias o para hostilizar a determinadas clases o universalidad de causantes; es decir, que se sustenten en bases objetivas y que justifiquen el tratamiento diferente entre una categoría y otra. Del mismo modo, referimos el criterio jurisprudencial 389615, que establece los elementos esenciales para la tributación como sujeto, objeto, base, tasa o tarifa y época de pago, que deben estar expresamente consignados en la ley (SJF, 1976) y obliga a la autoridad a no realizar actos que no estén previstos en una disposición legal anterior.

De acuerdo con la jurisprudencia que se refiere al principio de equidad, y siendo el catastro un ente que proporciona los elementos para el cobro de impuestos inmobiliarios, por analogía y atendiendo los dictados jurisprudenciales, se determinaron las clasificaciones para el suelo urbano y las categorías para las construcciones, con la finalidad de cumplir el precitado principio de equidad, considerando a los iguales y a los desiguales en la observancia del poder adquisitivo de la sociedad. 
De esta manera, el instructivo de valuación del estado de Sinaloa proporciona los elementos para determinar los valores catastrales, cuyo Valor Unitario de Suelo (calle o tramo de calle, zona, región o subregión) es determinado con base en la disponibilidad y las características urbanas relacionadas con la infraestructura y los servicios públicos; para establecer los valores de construcción se utiliza el análisis de precios unitarios que determinan el valor físico.

En la elaboración de los Avalúos Catastrales se hace uso del Instructivo de Valuación, que contiene el conjunto de normas que regulan el procedimiento para valorar las características del terreno y de la construcción, a fin de clasificarlos y asignarles los valores unitarios.

Sin embargo, a pesar de contar con el Instructivo de Valuación, una Ley del Catastro y su respectivo reglamento, el presente estudio nos muestra en sus resultados que, al obtener el valor catastral, los diferentes inmuebles considerados no cumplen con el principio de equidad, a pesar de que jurídicamente este valor debe ser la base para el pago de los impuestos inmobiliarios.

El trabajo sólo considera en su análisis los siguientes tipos de vivienda: de la Moderna 2 (M2) -o más económica- a la Moderna 7 (M7) -que es la más onerosa-, clasificadas en las categorías catastrales establecidas en el Instructivo de Valuación Catastral, como se observa en la figura 1.

\section{Figura 1 \\ Tipos de vivienda, definidos por sus especificaciones de construcción (particularmente acabados)}

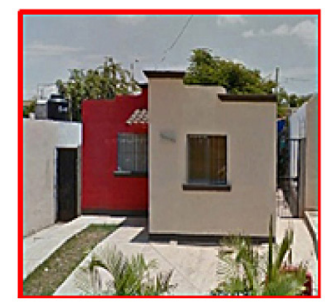

Vivienda tipo M2

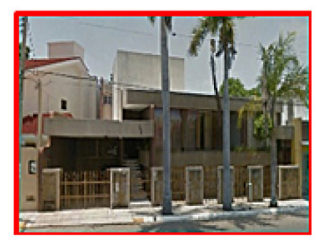

Vivienda tipo M5

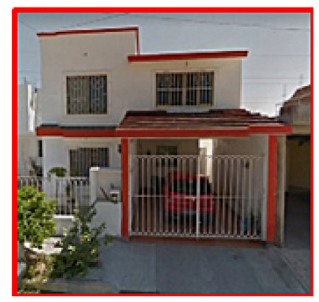

Vivienda tipo M3

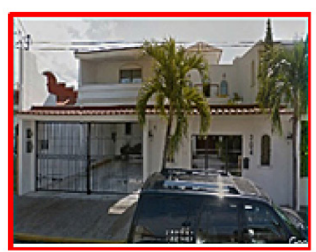

Vivienda tipo M6

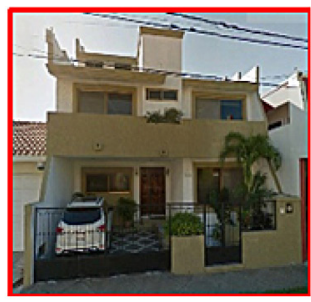

Vivienda tipo M4

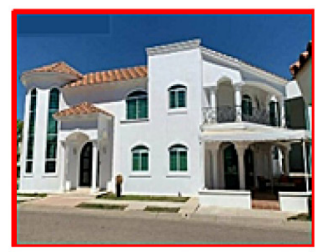

Vivienda tipo M7

Fuente: imágenes obtenidas de la plataforma de Google Earth Pro 2018. 


\section{Valor catastral y valor de mercado}

El valor catastral es netamente administrativo, obtenido mediante procedimientos objetivos para cada inmueble y está formado por el valor comercial de suelo y el valor físico de la construcción; tiene una participación directa en el pago de impuestos, pero también es usado con finalidades expropiatorias, impuestos sobre el patrimonio, de transmisión, entre otros (Roca, 1986).

Conceptualmente el valor de mercado es el precio más probable con el cual un bien inmueble podría ser vendido en un mercado bajo un funcionamiento normal, sin presiones o eventualidades que lo afecten de forma atípica con respecto a su oferta y su demanda. Tiene como características un vendedor y un comprador que actúan por su propia cuenta e interés económico y ambas partes se encuentran informadas. Asimismo, el bien a tratar debe durar un tiempo razonable en oferta para un mercado abierto y que no existan acciones abusivas por parte del vendedor; este valor se usa en el presente estudio sólo como una referencia máxima de valor.

En su artículo $6^{\circ}$ fracción LX, al referirse al valor catastral (CELSS, 2016), la Ley de Catastro del Estado de Sinaloa establece que para el terreno debe ser el valor comercial y para la construcción el valor físico que tenga el predio a la fecha de su avalúo; esto está establecido por normativas técnicas específicas para su fin, como el pago de impuestos inmobiliarios (Garcia-Almirall, 2007).

\section{Metodología}

\section{1. Área de estudio}

El espacio geográfico que comprende el área de estudio es la ciudad de Mazatlán, Sinaloa (México), ubicada al noroeste de la república mexicana, segunda en importancia en la entidad. Cuenta con una superficie municipal de $3068 \mathrm{~km}^{2}$ y un área urbana de 78,953 km² (Inegi, 2017); tiene 146,636 viviendas habitadas (Inegi, 2017), distribuidas en 187 fraccionamientos, 141 colonias, cinco unidades habitacionales y un conjunto habitacional, sumando un total de 334 asentamientos regulares registrados en el Plan Director de Desarrollo Urbano de la Ciudad de Mazatlán, Sinaloa (PDDUCM, 2013), que contiene en su parque inmobiliario todas las categorías de vivienda establecidas en el instructivo de valuación del estado de Sinaloa. 


\subsection{Proceso de la información}

Inicialmente se procedió a hacer un sondeo mediante el método Delphi, que es un recurso multicriterio cualitativo para toma de decisiones, ${ }^{1}$ utilizado cuando no se dispone de mucha información; para ello, el universo se conformó con expertos sobre la problemática a plantear (Torrado y Reguant, 2016), considerados con base en su experiencia profesional en el área de la valuación inmobiliaria, miembros de los distintos colegios de valuadores, a quienes se les aplicó una encuesta continua limitada (excelente, muy bueno, bueno, regular y malo).

El Instituto Catastral de la entidad elabora una propuesta de valores que envía a los ayuntamientos para su discusión, por lo que las preguntas de la encuesta se refieren a diversos aspectos sobre los valores catastrales que se deciden en la Junta Municipal de Catastro, la cual define, entre otros puntos, el valor unitario del terreno y el valor de la construcción, los criterios aplicados para los deméritos y el incremento de valor de acuerdo con el Instructivo de Valuación de Catastro, así como la delimitación de las Zonas de Valor, los Tramos de Calles del plano catastral y en general del plano catastral, los cuales posteriormente son publicados en el Periódico Oficial del Estado de Sinaloa (POES, 2016).

Después de realizadas las cuatro fases del método Delphi, como lo describe Astigarraga (2003), destaca la inconformidad de los expertos con respecto al catastro, ya que en la mayoría de sus respuestas manifestaron su desacuerdo con respecto a la implementación de los valores catastrales por parte de las juntas municipales de Catastro y lo publicado en el Periódico Oficial del Estado de Sinaloa con relación al mencionado valor catastral.

La encuesta fue aplicada a 106 profesionales de la valoración, cuyas respuestas y respectivos porcentajes pueden apreciarse en la tabla 1.

Habiendo recabado toda la información se obtuvo una media total de 2147 , tendencia regular de acuerdo con la escala establecida en el proceso de las decisiones que toma la Junta Municipal de Catastro y las tomadas por el Congreso del Estado con respecto al valor catastral. Asimismo, se observó que la categoría modal en cada una de las respuestas fe de malo $(40,43,47$ y $45 \%$ para las preguntas $1,2,6$ y 7 ) y regular (preguntas 3 , 4 y 5), esto determinó la moda (número que se presenta con más frecuencia en un conjunto de datos) en cadauna de las preguntas realizadas, la cual osciló entre malo y regular (pregnutas 3 y cuatro).

\footnotetext{
${ }^{1}$ Una decisión multicriterio es el conjunto de aproximaciones, métodos, modelos, técnicas y herramientas cuyo objetivo es mejorar la calidad integral de los procesos de decisión tanto de individuos como de sistemas, con el propósito de optimizar la efectividad, eficacia y eficiencia de los procesos de decisión e incrementar su conocimiento (Moreno-Jiménez, 1996).
} 
Si bien esta técnica es exploratoria y con pocos resultados estadísticos relevantes, resulta útil debido a la importancia del grupo seleccionado para el estudio; la encuesta nos muestra, en resumen, la opinión de los expertos (Varela et al., 2012), que arriban a un nivel de acuerdo de $70 \%$, aproximadamente, ubicándose entre malo y regular sobre la problemática en cuestión.

El uso del método Delphi es apropiado porque da prioridad a criterios generales para la evaluación de una investigación (Hartwich, 1998). En nuestro caso, los resultados de la investigación exploratoria avalan el interés por conocer las variables que componen al valor catastral y de qué manera sus componentes pueden ejercer una influencia sobre cada una de sus categorías; de esta manera, se convirtió en el motor motivacional para indagar el grado de inequidad existente en el pago de los tributos inmobiliarios.

Tabla 1

Total de respuestas y porcentajes obtenidos de los especialistas en valuación inmobiliaria

\begin{tabular}{|c|c|c|c|c|c|}
\hline $\begin{array}{r}\text { Calificación/Pregunta } \\
-\end{array}$ & Malo & Regular & Bueno & $\begin{array}{l}\text { Muy } \\
\text { Bueno }\end{array}$ & Excelente \\
\hline $\begin{array}{l}\text { 1. Junta Municipal } \\
\text { de Catastro }\end{array}$ & $42 / 40 \%$ & $22 / 21 \%$ & $18 / 17 \%$ & $14 / 13 \%$ & $10 / 9 \%$ \\
\hline $\begin{array}{l}\text { 2. Publicación POES } \\
\text { VUT }\end{array}$ & $46 / 43 \%$ & $23 / 22 \%$ & $17 / 16 \%$ & $10 / 9 \%$ & $10 / 9 \%$ \\
\hline $\begin{array}{l}\text { 3. Publicación POES } \\
\text { VUC }\end{array}$ & $30 / 28 \%$ & $48 / 45 \%$ & $15 / 14 \%$ & $8 / 8 \%$ & $5 / 5 \%$ \\
\hline $\begin{array}{l}\text { 4. Criterio para } \\
\text { Incrementos }\end{array}$ & $35 / 33 \%$ & $43 / 41 \%$ & $13 / 12 \%$ & $8 / 8 \%$ & $7 / 7 \%$ \\
\hline 5. Criterio para Deméritos & $35 / 33 \%$ & $44 / 42 \%$ & $14 / 13 \%$ & $7 / 7 \%$ & $6 / 6 \%$ \\
\hline $\begin{array}{l}\text { 6. Delimitación Zonas } \\
\text { de Valor }\end{array}$ & $50 / 47 \%$ & $38 / 36 \%$ & $11 / 10 \%$ & $5 / 5 \%$ & $2 / 2 \%$ \\
\hline $\begin{array}{l}\text { 7. Delimitación Tramos } \\
\text { de Calle }\end{array}$ & $48 / 45 \%$ & $42 / 40 \%$ & $10 / 9 \%$ & $5 / 5 \%$ & $1 / 1 \%$ \\
\hline$X=2.147$ & & & & & \\
\hline
\end{tabular}

Fuente: elaboración propia con base en el conteo realizado a las respuestas obtenidas en la encuesta aplicada a los especialistas en valuación.

Nota: POES VUT = publicación de los Valores Unitarios del Suelo en el Periódico Oficial del Estado de Sinaloa.

POES VUC = publicación de los Valores Unitarios de Construcción en el Periódico Oficial del Estado de Sinaloa. 


\subsection{Muestra y cálculo de la información}

La muestra consta de 176 viviendas por cada Categoría Catastral de Vivienda (de M2 a M7), tomada directamente de la base de datos de la Delegación Sur del Instituto Catastral del Estado de Sinaloa (ICES), de la cual se capturó la siguiente información (criterios): Clave Catastral, Categoría, Coordenadas X, Y, Z, Valor Catastral, M2 de Construcción (M2 C), M2 Unitario de Suelo (M2 S), Número de Habitaciones (NH), Número de Bańos (NB). Posteriormente se procedió a desglosar en criterios este valor catastral:

\begin{tabular}{|l|l|l|l|l|l|l|l|l|}
\hline \multicolumn{10}{|c|}{ Criterios Catastrales } \\
\hline Categoría & $\mathrm{M}^{2} \mathrm{C}$ & $\mathrm{M}^{2} \mathrm{~S}$ & $\mathrm{~N}_{\mathrm{H}}$ & $\mathrm{N}_{\mathrm{B}}$ & $\mathrm{V}_{\mathrm{UCD}}$ & $\mathrm{V}_{\mathrm{USD}}$ & $\mathrm{V}_{\mathrm{UCI}}$ & $\mathrm{V}_{\mathrm{USP}}$ \\
\cline { 2 - 9 } & $\mathrm{V}_{\mathrm{CRN}}$ & $\mathrm{F}_{\mathrm{D}}$ & $\mathrm{V}_{\mathrm{CP}}$ & $\mathrm{V}_{\mathrm{CoRN}}$ & $\mathrm{F}_{\mathrm{DC}}$ & $\mathrm{V}_{\mathrm{CCN}}$ & $\mathrm{V}_{\mathrm{CI}}$ & $\mathrm{V}_{\mathrm{CO}}$ \\
\hline
\end{tabular}

Valor Unitario de Construcción Decreto $\left(\mathrm{V}_{\mathrm{UCD}}\right)$, Valor Unitario de Suelo Decreto $\left(\mathrm{V}_{\mathrm{USD}}\right)$, Valor Unitario de Construcción ICES $\left(\mathrm{V}_{\mathrm{UCI}}\right)$, Valor Unitario de Suelo Promedio $\left(\mathrm{V}_{\mathrm{USP}}\right)$,Valor Catastral de Reposición Nuevo $\left(\mathrm{V}_{\mathrm{CRN}}\right)$, Factor de Deméritos $\left(\mathrm{F}_{\mathrm{D}}\right)$, Valor Catastral del Predio $\left(\mathrm{V}_{\mathrm{CP}}\right)$, Valor Construcción de Reposición Nuevo $\left(\mathrm{V}_{\mathrm{CoRN}}\right)$, Factor de Demérito a la Construcción $\left(\mathrm{F}_{\mathrm{DC}}\right)$, Valor Catastral de Construcción Neto $\left(\mathrm{V}_{\mathrm{CCN}}\right)$, Valor Catastral Inferido $\left(\mathrm{V}_{\mathrm{CI}}\right)$, Valor Catastral Oficial $\left(\mathrm{V}_{\mathrm{CO}}\right)$.

Para obtener el valor catastral, tal como lo especifican los artículos 6 y 34 de la Ley Catastral (POES, 2016), se toma en cuenta el Valor Comercial de Suelo más el Valor Físico de las Construcciones. Con respecto a los valores de mercado, la referencia del valor de suelo fue tomada del Libro Verde (AMPI, 2018). Se determinó la siguiente información de mercado a cada vivienda contenida en la muestra:

\begin{tabular}{|l|l|l|l|l|l|l|l|l|}
\hline \multicolumn{1}{|c|}{ Criterios de Mercado } \\
\hline Categoría & $\mathrm{V}_{\mathrm{USA}}$ & $\mathrm{V}_{\mathrm{USI}}$ & $\mathrm{V}_{\mathrm{UC}}$ & $\mathrm{V}_{\mathrm{MPA}}$ & $\mathrm{V}_{\mathrm{MC}}$ & $\mathrm{V}_{\mathrm{MPI}}$ & $\mathrm{V}_{\mathrm{MI}}$ & $\mathrm{V}_{\mathrm{MA}}$ \\
\hline
\end{tabular}

Valor Unitario de Suelo AMPI ( $\left.\mathrm{V}_{\text {USA }}\right)$, Valor Unitario de Suelo Inferido $\left(\mathrm{V}_{\mathrm{USI}}\right)$, Valor Unitario de Construcción $\left(\mathrm{V}_{\mathrm{UC}}\right)$, Valor Mercado del Predio AMPI $\left(\mathrm{V}_{\mathrm{MPA}}\right)$, Valor Mercado Construcción $\left(\mathrm{V}_{\mathrm{MC}}\right)$, Valor Mercado de Predio Inferido $\left(\mathrm{V}_{\mathrm{MPI}}\right)$, Valor Mercado Inferido $\left(\mathrm{V}_{\mathrm{MI}}\right)$, Valor Mercado $\operatorname{AMPI}\left(\mathrm{V}_{\mathrm{MA}}\right)$. 


\begin{tabular}{|l|l|l|l|l|l|}
\hline \multicolumn{7}{|c|}{ Criterios de Ratios } \\
\hline Categoría & $\mathrm{R}_{\mathrm{VCO} / \mathrm{VMA}}$ & $\mathrm{R}_{\mathrm{VCO} / \mathrm{VMI}}$ & $\mathrm{R}_{\mathrm{VCI} / \mathrm{VCO}}$ & $\mathrm{R}_{\mathrm{VMA} / \mathrm{VCI}}$ & $\mathrm{R}_{\mathrm{VMI} / \mathrm{VCI}}$ \\
\hline
\end{tabular}

Ratio Valor Catastral Oficial/ Valor Mercado AMPI ( $\left.\mathrm{R}_{\mathrm{VCO} / \mathrm{VMA}}\right)$, Ratio Valor Catastral Oficial/ Valor Mercado Inferido $\left(\mathrm{R}_{\mathrm{vCO} / \mathrm{VMI}}\right)$, Ratio Valor Catastral Inferido/Valor Catastral Oficial $\left(\mathrm{R}_{\mathrm{vCI} / \mathrm{vCO}}\right)$, Ratio Valor Mercado AMPI/Valor Catastral Inferido $\left(\mathrm{R}_{\mathrm{VMA} / \mathrm{VCI}}\right)$, Ratio Valor Mercado Inferido/ Valor Catastral Inferido $\left(\mathrm{R}_{\mathrm{VMI} / \mathrm{VCI}}\right)$.

Una vez obtenida la información de los criterios o variables para cada una de las viviendas de estudio, se implementó el método multicriterio CRITIC, original de Diakoulaki et al. (1995). Su nombre es el acrónimo de CRiteria Importance Through Intercriteria Correlation (importancia de criterios a través de su correlación).

Este método, acompañado por el ratio (IVSC, 2017), se convierte en la metodología Ratio+CRITIC y CRITIC+Ratio (Aznar y Guijarro, 2012), la cual se ubica en el terreno de los métodos comparativos, ya que permite calcular el valor de un activo mediante su contraste con otros activos comparables, de los cuales se conocen sus características y su valor (Aznar et al., 2011).

Partimos de la premisa de que los métodos objetivos obtienen información de datos observables (Jahan et al., 2012), por lo que el carácter imparcial de la base de datos garantiza la objetividad de este trabajo y por ende es importante para el resultado del proceso de ponderación, al no depender de criterios subjetivos.

Para el cálculo de la información, primeramente se seleccionó la categoría a valorar con base en la muestra adecuada. Se consideró que en ambas debían coincidir sus características físicas con las descritas en el Instructivo de Valuación del Estado de Sinaloa; asimismo, se debían conocer los valores y cada una de las características que las componen.

Posteriormente se desglosó el valor catastral en los criterios a emplear para el estudio, a fin de generar la Nueva Base de Datos (NBD). Este modelo se encontró dentro de las metodologías basadas en la comparación. Al tener conocimiento de los precios de cada una de las muestras comparables, fue viable obtener como resultado el valor sobre un sujeto de la categoría a tratar; en nuestro caso, se analizaron las categorías de vivienda catastrales M2, M3, M4, M5, M6 y M7.

Es fundamental tener bien definidos los criterios que se emplearán en la comparación, ya que por lógica cada una de las variables no puede tener la misma importancia al momento de determinar el valor catastral; por lo tanto, es primordial dar o asignar un valor objetivo al calcular una ponderación a cada criterio utilizado en la NBD. 
Gran parte de la problemática con respecto a la valoración de bienes inmuebles se encuentra en la subjetividad aplicada por los valuadores, ya que cuando se emiten juicios de valor, un mismo objeto puede ser ponderado de forma distinta por un grupo de profesionales, lo que genera inconsistencias en los valores aplicados, ya que como apuntan Yalcin y Ünlü (2017), muchas valuaciones dependen de las preferencias subjetivas.

Otros autores, entre ellos Diakoulaki et al. (1995) y Akçakanat et al. (2018), son más exigentes en este punto, pues señalan que no debe haber preferencia alguna al momento de asignar un valor; por el contrario, el propósito es obtener resultados objetivos que no tengan diferencias con el método a utilizar o con las variables que operen en él.

Para evitar esa subjetividad se usa la metodología CRITIC o método Diakoulaki, cuyo uso es recomendado para generar comparaciones realizadas sobre la base de múltiples ratios financieros en empresas, ya que clasifica objetivamente los criterios y no de una forma subjetiva y arbitraria (Li et al., 2018; Mu y Pereyra Rojas, 2017).

Lo anterior permite construir puntos de referencia sobre una situación financiera, pues define objetivamente los pesos de los criterios, de tal modo que los encargados de tomar decisiones no se apoyen sobre sus preferencias (Linares, 1999), lo cual facilita la toma de una decisión en la problemática del caso en cuestión sobre la importancia relativa de cada criterio y reduciendo la subjetividad.

El método CRITIC es aplicable en etapas de ajuste y, combinado con una metodología multicriterio, basada en información cuantitativa clara, demuestra la importancia relativa de cada uno de los criterios que nos ayudarán a tomar una mejor decisión. Se basa en el análisis de una matriz de datos de donde se extrae toda la información contenida en los criterios a evaluar (variables explicativas) (Akçakanat et al., 2018; Jahan et al., 2012; Yalçin y Ünlü, 2017). Con este análisis, apoyado en el ratio de valuación, se obtiene la primera forma Ratio+CRITIC, se normaliza cada variable explicativa con la finalidad de tener valores que oscilen entre 0 y 1 , lo que nos permitirá calcular sus ratios globales (Aznar et al., 2012) y con este ratio se calculó el valor por variable del sujeto en cuestión.

CRITIC está basado en el enfoque de la desviación estándar (Jahan $e t$ al., 2012), así que posteriormente se calcula ésta a cada variable; con su resultado, se calcula el Coeficiente de Correlación, usado comúnmente para medir la dependencia entre dos variables (Jahan et al., 2012), y posteriormente se calculan los coeficientes de correlación entre las variables.

La desviación estándar y el coeficiente de correlación de Pearson nos darán la información necesaria para obtener la ponderación relativa $(\mathrm{P})$ de cada una de las variables explicativas utilizando la siguiente expresión: 


$$
P_{j}=\sigma_{j} \cdot \sum_{k=1}^{m}\left(1-r_{j k}\right)
$$

Donde: $P_{j}$ es el peso relativo (cantidad de información contenida en la variable) $j, \sigma_{j}$ es la desviación estándar de la variable $j$ y $r_{j k}$ es el coeficiente de correlación de los criterios $j$ y $k$.

Como paso final se normaliza la información para obtener pesos o ponderaciones objetivas $(W)$, mediante la siguiente ecuación:

$$
W_{j}=\frac{P_{j}}{\sum_{k=1}^{m} P_{k}}
$$

Donde $W_{j}$ es el peso objetivo de la variable $j, P_{j}$ es el peso relativo de la variable y $\stackrel{P}{P}_{k}$ es la sumatoria del peso de las variables $j$.

Como se comentó, este método es comparativo; en nuestro caso, su característica es que toda la información referida a las ponderaciones y al valor a calcular se obtienen de la base de datos de los inmuebles registrados con características similares (Roca, 1986).

La información utilizada proviene de la BD de catastro y del Libro Verde (AMPI, 2018). De esta manera se obtiene una BD comparable donde se conoce el valor, tal como se ha registrado, y una serie de datos para las variables que comprenden el total de la información, lo cual genera una matriz. Toda esta información es necesaria para calcular las ponderaciones y el valor de un inmueble, paso importante para llegar al cumplimiento del objetivo de nuestro estudio: demostrar la inequidad del valor catastral; ya obtenidas las ponderaciones y usando el valor calculado por variable de los ratios, se obtiene el valor del sujeto o de los sujetos en cuestión. A este procedimiento se le conoce como Ratio+CRITIC.

$$
V_{x}=w_{v 1} \times \$_{R V 1}+w_{v 2} \times \$_{R V 2}+\cdots w_{v n} \times \$_{R V n}
$$

Donde $V_{x}$ es el valor del sujeto, $w_{v 1}$ corresponde al valor del peso de la variable, $\$_{R V 1}$ se refiere al valor en $\$ M X N$, obtenido del ratio de la variable.

De la misma manera que el proceso anterior, a partir de la desviación estándar y del coeficiente de correlación se obtienen los pesos de cada variable mediante la ecuación 2 , para posteriormente conseguir la pon- 
deración individual de cada comparable establecida en la NBD; así, tendremos un peso individual por cada inmueble.

$$
w_{c}=w_{v c 1} \times f_{n v 1}+w_{v c 2} \times f_{n v 2}+\cdots w_{v c n} \times f_{n v n}
$$

Donde $W_{c}$ corresponde al peso del comparable, $W_{v c}$ se refiere al peso de la variable del comparable y $f_{n v}$ es el factor normalizado de la variable.

De esta manera, al conocer los precios de los comparables y sus pesos, se obtiene el ratio global, que nos servirá para calcular el valor de cada inmueble seleccionado como sujeto de obtención de valor. Conforme a lo establecido por Aznar y Guijarro (2012), utilizando el ratio global multiplicándolo por la ponderación individual del inmueble se obtiene su valor. Este proceso es conocido como CRITIC+Ratio.

Obtenidas las ponderaciones en relación con la NBD, mediante el uso de CRITIC, se procedió a calcular los valores catastrales en $\$ M X N$ de una muestra de 26 viviendas, que fueron analizadas con base en los modelos Ratio+CRITIC y CRITIC+Ratio antes descritos. Se compararon los valores obtenidos entre categorías (M2-M7) para verificar la homogeneidad entre ellas, mediante el Análisis de Varianza (ANDEVA) de doble vía con prueba post hoc de Holm-Šidák, tomando como valor para significancia estadística de $\mathrm{p}<0.01$. Al mismo tiempo se analizaron las diferencias estadísticas entre los pesos y ratios obtenidos utilizando ANDEVA de doble vía con prueba post hoc de Holm-Šidák, utilizando un valor para significancia estadística de $\mathrm{p}<0.01$.

\section{Resultados y discusión}

\subsection{Ponderaciones: catastrales, mercado AMPI y de ratios}

En el análisis realizado para obtener las 29 ponderaciones, correspondientes a las variables obtenidas con el uso del Método CRITIC y utilizando ANDEVA de doble vía, con la prueba post hoc de comparaciones múltiples de Holm-Šidák con $99 \%$ de significancia, se obtuvieron para las seis categorías catastrales que van desde la Categoría M2 a la Categoría M7 referidas a vivienda en el ICES, 16 datos referentes a información catastral, siete datos con información de mercado y seis datos referidos a los ratios obtenidos. El desarrollo del proceso se detalla a continuación.

En las ponderaciones catastrales que se muestran en la tabla 2, las variables $\mathrm{M}^{2} \mathrm{C}, \mathrm{M}^{2} \mathrm{~S}, \mathrm{~N}_{\mathrm{H}}, \mathrm{N}_{\mathrm{B}}, \mathrm{V}_{\mathrm{USD}}, \mathrm{V}_{\mathrm{CRN}}, \mathrm{F}_{\mathrm{D}}, \mathrm{V}_{\mathrm{CP}}, \mathrm{V}_{\mathrm{CoRN}}, \mathrm{F}_{\mathrm{DC}}, \mathrm{V}_{\mathrm{CCN}}, \mathrm{V}_{\mathrm{CI}}$ y $V_{C O}$ presentan diferencias significativas en sus ponderaciones $(\mathrm{p}<0.01)$ al comparar cada categoría catastral de vivienda con cada una de las 
variables seleccionadas. La ponderación de la variable $\mathrm{V}_{\mathrm{UCI}}$ en la categoría M5 tiene diferencia significativa de $(\mathrm{p}<0.01)$. Por último, las variables $\mathrm{V}_{\text {UCD }} \mathrm{y} \mathrm{V}_{\text {USP }}$ no tuvieron diferencias significativas.

En las ponderaciones de mercado AMPI (tabla 3 ) tenemos las variables $\mathrm{V}_{\text {USA }}, \mathrm{V}_{\text {USI }}, \mathrm{V}_{\text {MC}}, \mathrm{V}_{\text {MPA, }}, \mathrm{V}_{\text {MPI, }}, \mathrm{V}_{\text {MI }}$ y $\mathrm{V}_{\text {MA }}$; al comparar las seis categorías de inmuebles entre sí por cada variable se encontraron para todas ellas marcadas diferencias significativas $(\mathrm{p}<0.01)$. Al comparar la ponderación, la variable $V_{\text {MPA }}$ presentó una pequeña diferencia solamente en las categorías

\section{Tabla 2}

\section{Temperaturas 1}

\begin{tabular}{|c|c|c|c|c|c|c|c|c|c|c|c|c|c|c|c|c|}
\hline \multicolumn{17}{|c|}{ Ponderación Porcentual (\%) de la Información Catastral } \\
\hline Categoría & $\mathbf{M}^{2} \mathrm{C}$ & $\mathbf{M}^{2} \mathrm{~S}$ & $\mathrm{~N}_{\mathrm{H}}$ & $\mathrm{N}_{\mathrm{B}}$ & $V_{\text {UCD }}$ & $V_{\text {LSD }}$ & $V_{\mathrm{LCI}}$ & Vtsp & $V_{\mathrm{CRN}}$ & $F_{\mathbf{D}}$ & $V_{C P}$ & $V_{\text {CORN }}$ & $\mathrm{F}_{\mathrm{DC}}$ & $\mathrm{V}_{\mathrm{CCN}}$ & $\mathrm{V}_{\mathrm{Cl}}$ & $v_{\mathrm{CO}}$ \\
\hline $\begin{array}{c}\text { Casa Tipo } \\
\text { M2 }\end{array}$ & $3.58 \%$ & $4.29 \%$ & $1.81 \%$ & $1.83 \%$ & $0.00 \%$ & $2.89 \%$ & $0.00 \%$ & $0.00 \%$ & $3.46 \%$ & $4.42 \%$ & $7.77 \%$ & $3.58 \%$ & $7.78 \%$ & $6.86 \%$ & $5.20 \%$ & $5.45 \%$ \\
\hline $\begin{array}{c}\text { Casa Tipo } \\
\text { M3 }\end{array}$ & $3.97 \%$ & $4.12 \%$ & $1.52 \%$ & $1.52 \%$ & $0.00 \%$ & $2.94 \%$ & $0.00 \%$ & $0.00 \%$ & $3.61 \%$ & $2.21 \%$ & $7.49 \%$ & $3.97 \%$ & $5.19 \%$ & $6.40 \%$ & $5.09 \%$ & $6.33 \%$ \\
\hline $\begin{array}{c}\text { Casa Tipo } \\
\text { M4 }\end{array}$ & $3.29 \%$ & $3.69 \%$ & $1.25 \%$ & $1.24 \%$ & $0.00 \%$ & $3.38 \%$ & $0.00 \%$ & $0.00 \%$ & $3.00 \%$ & $5.58 \%$ & $4.95 \%$ & $3.29 \%$ & $6.81 \%$ & $4.68 \%$ & $3.60 \%$ & $3.73 \%$ \\
\hline $\begin{array}{c}\text { Casa Tipo } \\
\text { M5 }\end{array}$ & $3.59 \%$ & $3.89 \%$ & $1.32 \%$ & $1.32 \%$ & $0.00 \%$ & $2.57 \%$ & $0.04 \%$ & $0.00 \%$ & $3.35 \%$ & $5.99 \%$ & $5.17 \%$ & $3.59 \%$ & $7.16 \%$ & $4.23 \%$ & $3.21 \%$ & $3.00 \%$ \\
\hline $\begin{array}{c}\text { Casa Tipo } \\
\text { M6 }\end{array}$ & $3.44 \%$ & $3.51 \%$ & $3.33 \%$ & $3.33 \%$ & $0.00 \%$ & $1.05 \%$ & $0.00 \%$ & $0.00 \%$ & $3.13 \%$ & $8.54 \%$ & $3.74 \%$ & $3.44 \%$ & $10.58 \%$ & $8.94 \%$ & $6.93 \%$ & $6.80 \%$ \\
\hline $\begin{array}{c}\text { Casa Tipo } \\
\text { M7 }\end{array}$ & $4.14 \%$ & $6.53 \%$ & $2.92 \%$ & $2.92 \%$ & $0.00 \%$ & $3.12 \%$ & $0.00 \%$ & $0.00 \%$ & $3.93 \%$ & $1.92 \%$ & $6.74 \%$ & $4.14 \%$ & $2.62 \%$ & $4.43 \%$ & $4.11 \%$ & $3.97 \%$ \\
\hline \multicolumn{5}{|c|}{ Valor máximo } & \multicolumn{6}{|c|}{ Valor intermedio } & \multicolumn{6}{|c|}{ Valor mínimo } \\
\hline
\end{tabular}

Fuente: elaboración propia con base en las ponderaciones porcentuales calculadas por el método CRITIC en Microsoft Excel 2010.

\section{Tabla 3}

\section{Temperaturas 2}

\begin{tabular}{|c|c|c|c|c|c|c|c|c|}
\hline \multicolumn{9}{|c|}{ Ponderación Porcentual (\%) de la Información AMPI (Mercado) } \\
\hline Categoría & VUSA & $\mathbf{V}_{\text {USI }}$ & $\mathbf{V}_{\mathrm{UC}}$ & $\mathbf{V}_{\text {MPA }}$ & $\mathbf{V}_{\mathrm{MC}}$ & $\mathbf{V}_{\text {MPI }}$ & $\mathbf{V}_{\text {MI }}$ & $\mathbf{V}_{\text {MA }}$ \\
\hline $\begin{array}{c}\text { Casa Tipo } \\
\text { M2 }\end{array}$ & $2.11 \%$ & $3.11 \%$ & $0.00 \%$ & $5.12 \%$ & $6.13 \%$ & $6.15 \%$ & $3.53 \%$ & $3.20 \%$ \\
\hline $\begin{array}{c}\text { Casa Tipo } \\
\text { M3 }\end{array}$ & $2.42 \%$ & $3.25 \%$ & $0.00 \%$ & $5.35 \%$ & $8.53 \%$ & $4.04 \%$ & $3.30 \%$ & $3.70 \%$ \\
\hline $\begin{array}{c}\text { Casa Tipo } \\
\text { M4 }\end{array}$ & $2.30 \%$ & $4.34 \%$ & $0.00 \%$ & $4.19 \%$ & $10.54 \%$ & $4.08 \%$ & $2.89 \%$ & $3.04 \%$ \\
\hline $\begin{array}{c}\text { Casa Tipo } \\
\text { M5 }\end{array}$ & $2.80 \%$ & $4.67 \%$ & $0.00 \%$ & $5.37 \%$ & $11.97 \%$ & $4.41 \%$ & $3.21 \%$ & $3.17 \%$ \\
\hline $\begin{array}{c}\text { Casa Tipo } \\
\text { M6 }\end{array}$ & $1.35 \%$ & $2.63 \%$ & $0.00 \%$ & $3.68 \%$ & $7.14 \%$ & $3.73 \%$ & $2.97 \%$ & $3.04 \%$ \\
\hline $\begin{array}{c}\text { Casa Tipo } \\
\text { M7 }\end{array}$ & $4.19 \%$ & $4.78 \%$ & $0.00 \%$ & $5.88 \%$ & $9.33 \%$ & $6.06 \%$ & $3.80 \%$ & $3.81 \%$ \\
\hline \multicolumn{3}{|c|}{ Valor máximo } & \multicolumn{3}{|c|}{ Valor intermedio } & \multicolumn{3}{|c|}{ Valor mínimo } \\
\hline
\end{tabular}

Fuente: elaboración propia con base en las ponderaciones porcentuales. Calculadas por el método CRITIC en Microsoft Excel 2010 
$\mathrm{M} 3$ vs $\mathrm{M} 5$. En el caso de la variable $\mathrm{V}_{\mathrm{UC}}$, en ninguna de sus comparaciones se encontraron diferencias significativas.

En la tabla 4 se muestran las ponderaciones de las variables $\mathrm{RVCO} /$ VMA, RVCO/VMI, RVCI/VCO, RVMA/VCI, RVMI/VCI con diferencias significativas en todas las categorías.

\section{Tabla 4}

\section{Temperaturas 3}

\begin{tabular}{|c|c|c|c|c|c|}
\hline \multicolumn{6}{|c|}{ Ponderación Porcentual (\%) de la Información de los Ratios } \\
\hline Categoría & $\mathbf{R}_{\mathrm{VCO} / \mathrm{VMA}}$ & $\mathbf{R}_{\mathrm{VCO} / \mathrm{VMI}}$ & $\mathbf{R}_{\mathrm{VCl} / \mathrm{VCO}}$ & $\mathbf{R}_{\mathrm{VMA} / \mathrm{VCI}}$ & $\mathbf{R}_{\text {VMI/VCI }}$ \\
\hline $\begin{array}{c}\text { Casa Tipo } \\
\text { M2 }\end{array}$ & $2.77 \%$ & $2.69 \%$ & $1.49 \%$ & $2.38 \%$ & $2.41 \%$ \\
\hline $\begin{array}{c}\text { Casa Tipo } \\
\text { M3 }\end{array}$ & $3.32 \%$ & $3.15 \%$ & $1.48 \%$ & $3.59 \%$ & $3.53 \%$ \\
\hline $\begin{array}{c}\text { Casa Tipo } \\
\text { M4 }\end{array}$ & $5.00 \%$ & $4.83 \%$ & $0.99 \%$ & $4.73 \%$ & $4.57 \%$ \\
\hline $\begin{array}{c}\text { Casa Tipo } \\
\text { M5 } \\
\end{array}$ & $3.52 \%$ & $3.66 \%$ & $1.10 \%$ & $3.67 \%$ & $4.01 \%$ \\
\hline $\begin{array}{c}\text { Casa Tipo } \\
\text { M6 }\end{array}$ & $1.82 \%$ & $1.93 \%$ & $0.64 \%$ & $2.06 \%$ & $2.24 \%$ \\
\hline $\begin{array}{c}\text { Casa Tipo } \\
\text { M7 }\end{array}$ & $2.10 \%$ & $2.13 \%$ & $0.98 \%$ & $2.61 \%$ & $2.84 \%$ \\
\hline \multicolumn{2}{|c|}{ Valor maximo } & \multicolumn{2}{|c|}{ Valor intermedio } & \multicolumn{2}{|c|}{ Valor Minimo } \\
\hline
\end{tabular}

Fuente: elaboración propia con base en las ponderaciones porcentuales, calculadas por el método CRITIC en Microsoft Excel 2010.

\subsection{Precios}

Los precios arrojados por Ratio+CRITIC y CRITIC+Ratio generan un modelo de cálculo estandarizado de los valores, tomando en cuenta las ponderaciones parciales de cada variable; para las Categorías Catastrales de Vivienda M2 a M7 se analizaron 26 viviendas, de las cuales se conocía su valor catastral oficial y su valor de mercado, según datos del AMPI.

$\mathrm{Al}$ analizar los precios obtenidos utilizando ANDEVA de doble vía, con la prueba post hoc de comparaciones múltiples de Holm-Šidák con 99\% de significancia, cada una de las viviendas seleccionadas acusó un comportamiento homogéneo en los valores de cada categoría; es posible observar cambios entre categorías, porque se obtienen valores en $\$ \mathrm{MXN}$ y es normal que una vivienda de categoría M2 sea más económica que una vivienda de categoría M7.

El análisis de comparaciones múltiples muestra dicha homogeneidad entre las metodologías de valoración RATIO+CRITIC, CRITIC+RATIO 
y los valores catastrales oficiales; sin embargo, entre los valores catastrales inferidos, valor comercial AMPI y valor comercial inferido se encontraron diferencias significativas en las categorías catastrales de vivienda M5, M6 y M7 ( $<<0.01)$ por cada uno de los valores analizados (gráfica 1).

\section{Gráfica 1}

\section{Precio en $\$ M X N$ por cada categoría catastral de vivienda}

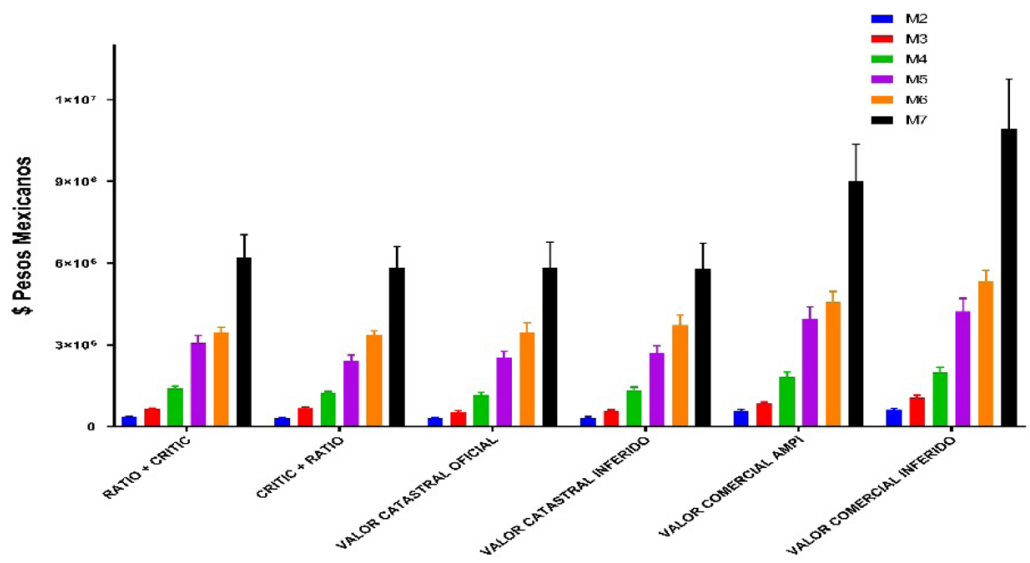

Fuente: elaboración propia con base en la información obtenida de valores de precios en \$MXN de la base de datos obtenida en el ICES, zona sur y el AMPI, en el software Graphpad Prism 7.0.

Durante el análisis multicriterio la variabilidad encontrada en las ponderaciones calculadas para cada variable tiene una influencia final en el precio calculado para las propiedades analizadas. Si las ponderaciones fueran homogéneas, encontraríamos proporcionalidades homogéneas entre las categorías al realizar ratios de valores calculados por las diferentes metodologías. Sin embargo, se encontraron diferencias muy significativas en los ratios de valores entre ellas (gráfica 2).

Los datos fueron analizados mediante ANDEVA de doble vía con la prueba post hoc de comparaciones múltiples de Holm-Šidák con 95\% de confiabilidad; se encontró que hubo diferencias significativas entre los ratios de las categorías M3 y M7 en RATIO+CRITIC/ \$CATASTRAL OFICIAL $(\mathrm{p}<0.05)$. Entre M3 y M4, M5 ( $\mathrm{p}<0.05)$, M3 y M7 $(\mathrm{p}<0.01)$ en CRITIC+RATIO/\$CATASTRAL OFICIAL. Entre M2 y M4, M5, M6 y M7 (p<0.01) al comparar \$COM AMPI/\$CAT INFERIDO, y entre M2 y M7 y entre M3 y M5 $(\mathrm{p}<0.01)$ al comparar $\$$ COM INFERIDO/\$CAT INFERIDO.

Las diferencias significativas en los ratios confirman la falta de homogeneidad derivada de las ponderaciones desiguales, encontradas al realizar 


\section{Gráfica 2}

\section{Ratios de valores calculados}

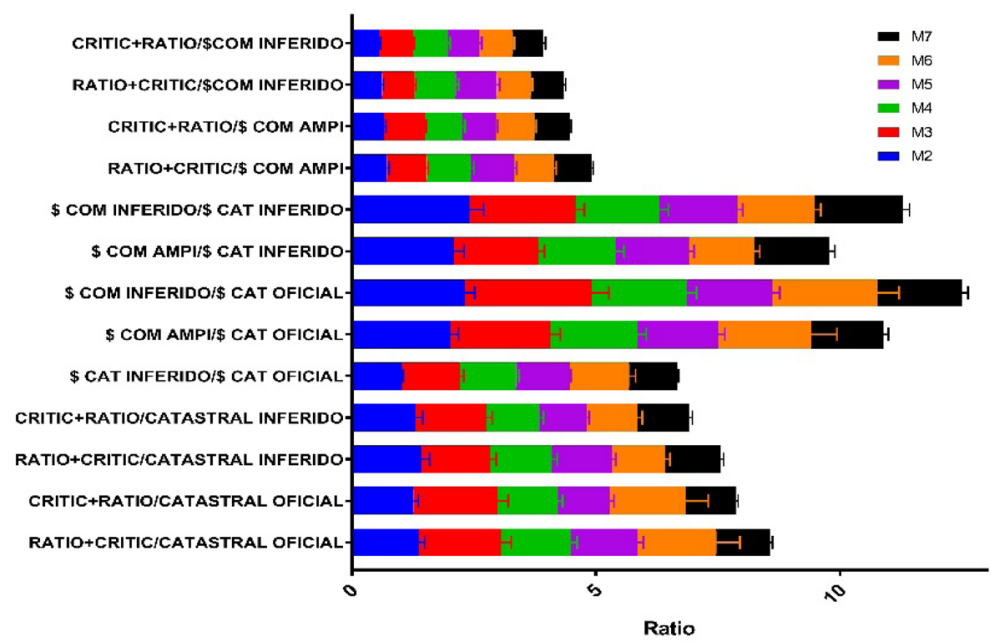

Fuente: elaboración propia con base en los valores medios de los ratios calculados en la base de datos obtenidos en ICES y el AMPI, en el software Graphpad Prism 7.0.

el análisis multicriterio. Si las ponderaciones de las variables fuesen homogéneas, debería esperarse homogeneidad en las magnitudes de los ratios de precios calculados entre cada categoría analizada. Sin embargo, se observa que al realizar ratios entre los precios calculados con metodología multicriterio estandarizada (Ratio+CRITIC y CRITIC+Ratio), existen discrepancias estadísticamente significativas $(\mathrm{p}<0.05)$ entre categorías en los valores catastrales oficiales y se observa un incremento en categoría M3 y M6, así como una disminución significativa en M7, al compararse con las categorías M2, M4 y M5.

Si entendemos que los precios obtenidos a través de la metodología multicriterio son valores en los que las ponderaciones desiguales se intentan normalizar para minimizar la variabilidad, las desigualdades que se observan, principalmente en la obtención de los valores catastrales oficiales, son las responsables de las diferencias significativas encontradas en estos ratios. Entonces, los valores catastrales afectados derivan en efectos posteriores en el pago de los impuestos.

Con el objeto de tener una visión más amplia de lo que se pretende con este estudio, se revisaron las diferentes metodologías usadas en la valoración de inmuebles; así, tenemos los modelos econométricos, de los cuales destacamos: el Valor Analógico Bursátil (Caballer y Moya, 1998) y el presentado por Martínez (1998) en la Valoración Inmuebles Rústicos. 
Ambos métodos fueron examinados debido a la necesidad de obtener amplia información, tanto de valor como de variables explicativas a utilizar; asimismo, por la exigencia de precisión, era imperativo que fueran numerosas. Sin embargo, las fuentes nos obligan a utilizar información subjetiva al quedar al arbitrio de los valuadores, debido a las características del bien o bienes a analizar.

Humarán (2010) y Aguiló (2002) sugieren el uso inicial del Modelo de Precios Hedónicos (Ridker y Henning, 1967), mediante el cual se utiliza la variable de contaminación ambiental que modifica el valor de las viviendas. Destacan el uso de atributos endógenos (internos) y exógenos (externos), que tienden a ser interpretados subjetivamente, lo cual se busca evitar en este estudio. Asimismo, Calatrava (2003) y Núñez et al. (2008) subrayan las dificultades en el uso de método de precios hedónicos debido a que se enfoca en la necesidad de obtener un gran número de variables y la subjetividad que ello implica.

Todos los métodos coinciden en un punto: dar certidumbre a la información empleada en los cálculos (Aznar y Guijarro, 2012); por eso se busca la objetividad de la información con el propósito de reducir o eliminar todo sesgo proveniente de la apreciación subjetiva; para este fin se encuentran las Metodologías Multicriterio para la toma de decisiones. Entre estos métodos se encuentra el Modelo Analítico Jerárquico (AHP, por sus siglas en inglés), que a juicio de Olson (1988), Mu y Pereyra Rojas (2017), Moreno-Jiménez et al. (1998), Hartwich (1998) y Escobar y Moreno (1997) presenta dificultades e inconvenientes relacionados con la subjetividad, a pesar de ser el más utilizado.

Otros métodos que abordan el problema de la subjetividad son MAUT y ELECTRE, descritos por Berumen y Llamazares Redondo (2007); el mismo caso sucede con el método PROMETHEE, mediante el cual el usuario puede colocar valores arbitrarios a las ponderaciones, tal como lo mencionan Brans y Mareschal (2005), o el método TOPSIS, aplicado al análisis de tractores agrícolas por García-Alcaraz y Noriega (2008), que nos habla sobre la subjetividad que interviene en el proceso de dicho método.

También encontramos el Método de Programación por Metas (GP), usado por Charnes y Cooper (1968), que tiene el inconveniente del sesgo de la subjetividad del operador del método. A su vez, en su estudio, LópezOspina y López (2010) señalan que obtener un valor real del objeto o de los objetos con el Método GP es complicado, ya que solamente se obtiene información parcial. Así, con esta subjetividad, este método es aplicado a la valoración inmobiliaria y de empresas por autores como Aznar et al. (2011) y Aznar y Guijarro (2012), entre otros. 
Las dificultades metodológicas anteriores han motivado la búsqueda de un método más objetivo (Diakoulaki et al., 1995; Alemi-Ardakani et al., 2016; Liu y Zhao, 2013), mediante el cual las ponderaciones son obtenidas de la misma base de datos descartando la percepción del investigador. Como lo establece Pellice (2008), el CRITIC o método Diakoulaki es exclusivo para el cálculo de ponderaciones y cuando se acompańa del ratio de valuación (Caballer y Aznar, 2004; Aznar y Guijarro, 2012) se obtienen valores inmobiliarios (Aznar et al., 2016; Aznar y Guijarro, 2012).

Al obtener la ponderación de las categorías catastrales analizadas mediante la metodología multicriterio Ratio+CRITIC y CRITIC+Ratio, encontramos diferencias entre el peso de las variables y la categoría catastral de la vivienda utilizada. Se observa la diferencia del peso o ponderación de cada variable referida a su categoría de manera ilustrativa en las tablas, donde se puede apreciar, mediante la siguiente escala de color, el identificador rojo para el valor más bajo, para valores intermedios el color amarillo y para valores estimados como altos, el color azul, que representan la importancia porcentual de la ponderación obtenida con respecto a la variable.

Con la Información Catastral en la tabla 2 se observa el comportamiento de algunas variables. Analizamos los valores obtenidos para la variable $\mathrm{M}^{2} \mathrm{~S}$, la categoría catastral de vivienda $\mathrm{M} 7$ tiene una importancia de $6.53 \%$, seguida de M2 con $4.29 \%$ y M3 con $4.12 \%$, entendiendo que éstas son las categorías cuya variable tuvo importancia alta; con respecto a M4, M5 y M6, la importancia obtenida en la variable fue media.

Con relación a la variable $\mathrm{V}_{\text {USD }}$, observamos que para la Categoría Catastral de vivienda M6 se obtuvo un valor de $1.05 \%$, seguida de M5 con $2.57 \%$. Las consideramos de importancia baja con respecto al resto de categorías (M2, M3, M4 y M7) porque sus porcentajes oscilan de $2.89 \%$ a $3.38 \%$, representando una importancia media. En la variable $\mathrm{F}_{\mathrm{D}}$ se obtuvieron los siguientes resultados: la categoría M6, 8.56\%, con importancia muy alta; M5, con 5.99\%; M4, con 5.58\% (importancia alta) y M2 con 4.42\% (importancia media); a su vez, las categorías M3 con $2.21 \%$ y M7 con $1.92 \%$, arrojaron importancia considerada como baja.

En la variable $\mathrm{V}_{\mathrm{CP}}$ se encontró lo siguiente: la categoría $\mathrm{M} 2,7.77 \%$ (muy alta); M3, 7.49\% y M7, 6.74\% (altas); M5, 5.17\% y M4, 4.95\% (media alta) y por último M6, con $3.74 \%$ (importancia media); la variable $\mathrm{F}_{\mathrm{DC}}$ arrojó a su vez: $\mathrm{M} 6,10.58 \%$ (muy alta importancia); $\mathrm{M} 2,7.78 \%$ y M5, 7.16\% (importancia alta); M4, 6.81\% y M3, 5.19\% (importancia media alta) y por último M7, con $2.62 \%$ (importancia baja).

Por otro lado, la variable $\mathrm{V}_{\mathrm{CCN}}$, en la categoría catastral de vivienda, presentó lo siguiente: M6, 8.94\% (importancia muy alta); M2, 6.86\% y 
M3, 6.40\% (importancia alta); M7 y M5, $4.43 \%$ y 4.23\%, respectivamente (importancia media); con referencia a las variables $\mathrm{V}_{\mathrm{CI}} \mathrm{y} \mathrm{V}_{\mathrm{CO}}$, las categorías M6, M3 y M2 obtuvieron una importancia alta, M7 una importancia media y las categorías M4 y M5 tendieron a una importancia baja.

Podemos advertir que en variables como $\mathrm{V}_{\mathrm{USP}}$ o $\mathrm{V}_{\mathrm{UCD}}$ se obtuvo una ponderación de $0 \%$ en todas las categorías catastrales de vivienda; en la variable $\mathrm{V}_{\text {CRN }}$ o en $\mathrm{V}_{\text {CoRN }}$ se observa un comportamiento más razonable en sus ponderaciones por categoría catastral, que exige el tipo de comportamiento que se debería observar al existir una razón acorde con el mercado y el catastro; sin embargo, la naturaleza de las características de los inmuebles nos obliga a considerar que siempre habrá variables más importantes que otras, pero en el caso de las categorías catastrales de vivienda, la importancia de cada variable debería de ser similar en todas las categorías.

Tomemos como ejemplo el siguiente: en la variable $\mathrm{F}_{\mathrm{D}}$ el ideal sería que todas las categorías catastrales de vivienda fueran de importancia alta, media o baja; entendiendo que podría haber variaciones, pero éstas deberían de ser mínimas. Lo mismo debería suceder con cada una de las variables que conforman el valor catastral con respecto a las categorías catastrales de vivienda.

En lo que respecta a las variables que conforman el mercado (AMPI), en la tabla 3 observamos que la variable $\mathrm{V}_{\text {USA }}$ en las categorías M2, M3, M4 y M5 presenta una importancia baja, pero en M6 obtuvo una ponderación muy baja y en $\mathrm{M} 7$ una ponderación media.

En el caso de la variable $\mathrm{V}_{\mathrm{MC}}$ se advierte una importancia alta; se puede notar que M4 (con 10.54\%) y M5 (con 11.97\%) son las categorías que concedieron mayor importancia a la variable, en comparación con M6, que tiene $7.14 \%$, y M2 con $6.13 \%$. A pesar de considerarse como altas, están entre 5 y 6 puntos porcentuales debajo de la más alta, por lo cual caemos en la misma problemática de no equidad, ya que se esperaría que sus ponderaciones fueran similares entre categorías catastrales y no con saltos abruptos en el valor de su ponderación en cada categoría con relación a la variable. Las variables $\mathrm{V}_{\text {MI }} \mathrm{y}_{\text {MA }}$ presentan ponderaciones similares en sus categorías correspondientes.

En la tabla 4 se alojan las ponderaciones sobre los ratios trabajados. En M4 se alcanzaron las más altas con respecto a las demás categorías, con excepción en el ratio $\mathrm{R}_{\mathrm{VCI} / \mathrm{VCO}}$, en el que todas las categorías resultaron con ponderación muy baja, por lo que se observa lo que afirma Madrid (2010): este método permite ubicar los atributos más importantes.

Nuestro enfoque busca la justicia tributaria, la cual se cumpliría si existiera correspondencia entre el valor catastral y el valor de mercado de 
los inmuebles en cuestión, como lo señala Llombart (1996). Por otra parte, con el método CRITIC no sólo es posible obtener la importancia o peso de un atributo, permitiendo con ello que se obtenga un precio o valor, sino que además nos ayuda a tomar una decisión en función de todas las variables según su importancia, de acuerdo con la contextualización de los modelos multicriterio de Carrasco y Martel (2017), pues con base en una ponderación podremos decidir una acción.

La ponderación de cada variable debería tener el mismo valor por cada categoría. No obstante, las diferencias encontradas entre cada categoría catastral nos permiten concluir que existe inequidad del valor catastral de vivienda con respecto al pago de los tributos inmobiliarios, como lo indica el título de nuestro trabajo.

\section{Conclusiones}

El propósito de este trabajo ha sido generar un análisis con la información emanada de la BD de la delegación sur del ICES, mediante el uso de métodos multicriterio. En este caso, con el método CRITIC y el Ratio de valuación, se calcularon las ponderaciones del valor catastral en cada categoría de vivienda del Instructivo de Valuación del ICES, donde se encontraron diferencias significativas entre ponderaciones de las variables que conforman el valor catastral y que a la postre influyen en la tributación inmobiliaria.

En esta parte del trabajo, una vez analizadas todas las variables, podemos responder nuestra pregunta central: ¿existe equidad en estos valores obtenidos? El procedimiento utilizado nos permitió obtener precios en $\$ M X N$ por vivienda de las categorías trabajadas, los cuales fueron estandarizados con base en el modelo generado, por lo cual arribamos a los siguientes resultados:

A simple vista se advierte que los porcentajes de las ponderaciones encontradas en las categorías analizadas (M2 a M7) presentan variaciones en el mismo criterio, lo que arroja que, para algunas categorías, las variables tengan más importancia que otras, afectando así desproporcionadamente el valor de cada vivienda, ya que si esta información fuera correcta desde su concepción en la $\mathrm{BD}$, la ponderación de cada variable por categoría catastral sería aproximadamente similar.

Se encontró que la Ponderación Porcentual de las variables (tablas 2, 3 y 4 ) tiene un efecto en el Valor Catastral, ya que a mayor grado porcentual de la ponderación habrá una influencia de aumento en el valor catastral y viceversa. Es importante recordar que la Categoría Catastral M2 es 
la más económica y aumenta progresivamente hasta la categoría M7 como la más onerosa.

Lo anterior lo abordamos en los siguientes ejemplos con las variables explicativas en las que intervienen el grueso de los valores en un inmueble, como son el suelo y la construcción. Veamos enseguida los siguientes análisis de las propias tablas:

1. En la variable Metros Cuadrados de Construcción $\left(\mathrm{M}^{2} \mathrm{C}\right)$, la Categoría M2 tiene una ponderación porcentual e influencia en el valor catastral de 3.58\%, mayor que M4 y M6.

2. En la variable Metros Cuadrados de Suelo $\left(M^{2} S\right)$, la ponderación porcentual e influencia en el valor catastral de la categoría M2 es de 4.29\%, mayor que M3, M4, M5, M6 y menor que M7.

3. Asimismo, observamos que en la variable Valor Catastral del Predio $\left(\mathrm{V}_{\mathrm{CP}}\right)$, la ponderación porcentual y la influencia de valor es mayor en las categorías M2 y M3, con 7.77\% y 7.49\%, respectivamente, seguido de M7 con 6.74\%; M4, M5 y M6 se constituyen como las más bajas.

4. En la variable Valor Catastral de Construcción Neto $\left(\mathrm{V}_{\mathrm{CCN}}\right)$, la ponderación porcentual y la influencia de valor es mayor en las categorías M2 y M3, con 6.86\% y $6.40 \%$ respectivamente, seguidas de M3, M4, M5 y M7 como las más bajas, y la M6 con 8.94\%, en este caso como la más alta.

Estos seńalamientos pueden ser repetitivos en cada una de las variables, pero con estos ejemplos se puede afirmar que, en lo general, estas diferencias significativas observables en las ponderaciones para las variables explicativas nos demuestran que éstas $-\mathrm{y}$ sobre todo las que tienen mayor importancia de valor como Valor Catastral del Predio $\left(\mathrm{V}_{\mathrm{CP}}\right)$ y de Valor Catastral de Construcción Neto $\left(\mathrm{V}_{\mathrm{CCN}}\right)$ - tienen su origen en la deficiente elaboración de la base de datos catastrales.

Así tenemos que las categorías M2 y M3, que son las más económicas, propias de los sectores sociales más vulnerables de la sociedad debido a su mayor ponderación porcentual, sufren un mayor impacto en el valor catastral, ya que se ubican en un rango de pago más alto al que le corresponde (tabla 5), lo cual implica una mayor tributación inmobiliaria. Un dato importante que observamos es que las viviendas con categorías M2 y M3 (clase baja y media baja) constituyen 78\% (análisis espacial mapa digital Inegi) del total de viviendas en la zona urbana de un total de 146,636 viviendas, que nos muestra cómo las clases sociales media, media alta y alta son beneficiadas al momento de pagar sus impuestos inmobiliarios.

También tenemos que en la categoría catastral M6, las variables Factor de Demérito (FD), Factor de Demérito de Construcción (FDC) y Valor Catastral de Construcción Neto (VCCN) presentan una mayor ponderación porcentual que la categoría $\mathrm{M} 7$, lo cual deriva en una mayor carga impositiva a la categoría M6, mientras que la M7 -que es la más onerosa- 
Tabla 5

Rangos de valores para el pago del impuesto predial

\begin{tabular}{|c|c|c|c|c|c|c|}
\hline \multicolumn{7}{|c|}{ TARIFA DEL IMPUESTO PREDIAL 2018} \\
\hline \multirow[b]{2}{*}{ No. } & \multicolumn{2}{|c|}{ VALOR DE LOS PREDIOS } & \multicolumn{2}{|c|}{ CONSTRUIDOS } & \multicolumn{2}{|c|}{ BALDÍOS } \\
\hline & $\begin{array}{c}\text { LIMITE } \\
\text { INFERIOR } \\
\text { EN PESOS } \\
\end{array}$ & $\begin{array}{c}\text { LIMITE } \\
\text { SUPERIOR } \\
\text { EN PESOS }\end{array}$ & $\begin{array}{c}\text { CUOTA FIJA } \\
\text { EN PESOS }\end{array}$ & $\begin{array}{c}\text { TASA AL } \\
\text { MILLAR } \\
\text { S/EXCED }\end{array}$ & $\begin{array}{l}\text { CUOTA FIJA } \\
\text { EN PESOS }\end{array}$ & $\begin{array}{l}\text { TASA AL } \\
\text { MILLAR } \\
\text { S/EXCED }\end{array}$ \\
\hline 1 & 0.01 & $43,851.30$ & 0.00 & 2.5 & 0.00 & 4.50 \\
\hline 2 & $43,851.31$ & $97,822.13$ & 109.64 & 2.55 & 197.34 & 5.05 \\
\hline 3 & $97,822.14$ & $209,136.99$ & 247.27 & 2.64 & 469.9 & 5.14 \\
\hline 4 & $209,137.00$ & $276,600.52$ & 541.16 & 2.77 & $1,042.07$ & 5.27 \\
\hline 5 & $276,600.53$ & $344,064.07$ & 728.04 & 2.95 & $1,397.61$ & 5.45 \\
\hline 6 & $344,064.08$ & $512,722.92$ & 927.07 & 3.31 & $1,765.30$ & 5.81 \\
\hline 7 & $512,722.93$ & $978,221.37$ & $1,485.34$ & 3.82 & $2,745.22$ & 6.32 \\
\hline 8 & $978,221.38$ & $1,484,197.92$ & $3,263.55$ & 4.28 & $5,687.18$ & 6.78 \\
\hline 9 & $1,484,197.93$ & $2,631,078.11$ & $5,429.14$ & 4.98 & $9,117.71$ & 7.48 \\
\hline 10 & $2,631,078.12$ & $5,397,083.28$ & $11,140.61$ & 5.37 & $17,696.38$ & 7.87 \\
\hline 11 & $5,397,083.29$ & EN ADELANTE & $25,994.07$ & 6.57 & $39,464.85$ & 9.07 \\
\hline
\end{tabular}

Fuente: Periódico Oficial del Estado de Sinaloa, núm. 158, viernes 15 de diciembre de 2017.

puede presentarse con un valor catastral que lo ubique en un rango menor para el pago de impuestos. Se vuelve a repetir el caso de que la clase social alta (M7) paga menos impuestos que la clase social media alta (M6).

La Ley de Hacienda Municipal para el Estado de Sinaloa establece las reglas para el cobro de impuestos, entre ellos el predial. Cada ańo, el Instituto Catastral del Estado de Sinaloa publica la tarifa del Impuesto Predial que reproducimos en la tabla 5, compuesta de 11 rangos de valores que contienen los valores de los predios, tanto construidos como baldíos con la cuota fija y tasa correspondiente.

El pago de impuestos se rige por rangos de valor y no por categorías, así que una categoría M2 puede llegar a tener un valor tal que se ubique en un rango igual o mayor a una categoría M3 o hasta M4, y por lo tanto tendrá una tributación mayor. Por otro lado, una categoría M6 puede tener un valor tal que la ubique en un rango de pago mayor que el que se tenga para una categoría M7. Esto es una negación de la justicia tributaria y una injusticia social: ejemplifica precisamente la existencia de inequidad en el pago de los impuestos.

Mediante el uso de esta metodología multicriterio se aporta una solución al ámbito de la valoración catastral, pues revela la importancia de las variables que componen el valor catastral en la localidad de Mazatlán, Sinaloa, bajo los criterios establecidos por el ICES; con esto se demuestra que se incumple el principio de equidad, ya que el valor expresado para cualquier inmueble establecido en las diferentes categorías catastrales de vivienda presenta diversas ponderaciones para una misma variable, 
afectando los valores que son aplicados para el cobro de los impuestos inmobiliarios.

Por ley, en los impuestos (en este caso el predial) se establece una correcta aplicación de la equidad en términos horizontales y verticales, pero si desde su concepción el valor catastral tiene divergencias, se incurre en la inequidad para los contribuyentes, ya que unos terminan pagando más que otros en dicha tributación y, por lo tanto, no se cumple con el espíritu de la bien llamada Justicia Tributaria.

La presente investigación no agota sin embargo las líneas de trabajo en lo referente a la valoración mediante el uso de metodología multicriterio enfocado al catastro. Esto se puede mejorar y afinar en nuevas investigaciones, mediante diferentes métodos que permitan comparar e incluir, tanto la información objetiva como subjetiva, así como desarrollar un método que genere un ajuste en las diferencias que pudiesen aparecer en los pesos de las variables para poder dar un mayor sustento al resultado de valor y una confianza en términos de igualdad tributaria a los contribuyentes, magno objetivo de la equidad fiscal.

\section{Fuentes consultadas}

Aguiló Segura, Paula María (2002), "El método de valoración de los precios hedónicos. Una aplicación al sector residencial de las islas baleares", tesis doctoral, Universidad de las Islas Baleares, Islas Baleares.

Akçakanat, Özen; Aksoy, Esra y Türker, Teker (2018), “Critic ve MDL Temelli Edas Yöntemí Íle TR-61 Bölgesi Bankalarinin Performans Degerlendimesi”, Journal of Süleyman Demirel University Institute of Social Sciences, 3 (32), Estambul, Süleyman Demirel University, pp. 1-24.

Alemi-Ardakani, Mohammad; Milani, Abbas; Yannacopoulos, Spiro y Shokouhi, Golnaz (2016), "On the effect of subjective, objective and combinative weighting in multiple criteria decision making: A case study on impact optimization of composites", Expert System With Applications, vol. 46, Nueva York, Elsevier, pp. 426438, doi: doi.org/10.1016/j.eswa.2015.11.003

AMPI (Asociación Mexicana de Profesionales Inmobiliarios) (2018), "Libro Verde Valuador de Propiedades", Mazatlán, AMPI. 
Astigarraga, Eneko (2003), "El Método Delphi”, Documento de Trabajo, San Sebastián, Universidad de Deusto.

Aznar Bellver, Jerónimo; Cayo Araya, Teodosio y Cevallos Varela, Diego (2016), Valoración de empresas. Métodos y casos prácticos para pequeñas y medianas empresas, Valencia, Universidad Politécnica de Valencia.

Aznar Bellver, Jerónimo; Cervelló, Roberto y García García, Fernando (2011), "Una alternativa multicriterio a la valoración de empresas: aplicación a las Cajas de Ahorro/A Multicriteria Alternative to Companies' Valuation: Application to a Spanish Savings Bank", Estudios de Economía Aplicada, 29 (1), Cádiz, Asociación de Economía Aplicada, pp. 1-16.

Aznar Bellver, Jerónimo; González Mora, Ronny; Guijarro, Francisco y López Perales, Arturo (2012), Valoración inmobiliaria: Métodos y Aplicaciones (España e Iberoamérica), Valencia, Universitat Politècnica de València.

Aznar Bellver, Jerónimo y Guijarro, Francisco (2012), Nuevos Métodos de Valoración, Modelos Multicriterio, Valencia, Universitat Politècnica de València.

Berumen, Sergio y Llamazares Redondo, Francisco (2007), "La utilidad de los métodos de decisión multicriterio en un entorno de competitividad creciente", Cuadernos de Administración, 20 (34), Bogotá, Pontificia Universidad Javeriana, pp. 65-87.

Brans, Jean-Pierre y Mareschal, Bertrand (2005), "Promethee Methods", en José Figueira, Salvatore Greco y Matthias Ehrogott, Multiple Criteria Decision Analysis: State of the Art, Surveys, vol. 78, Springer, Nueva York, pp. 163-186, doi: https://doi.org/10.1007/0387-23081-5_5

Caballer Mellado, Vicente y Aznar Bellver, Jerónimo (2004), "Metodología multicriterio aplicada a la valoración agraria", Revista Española de Estudios Agrosociales y Pesqueros, núm. 203, Madrid, Ministerio de Agricultura, Pesca y Alimentación, pp. 35-47.

Caballer Mellado, Vicente y Moya, Ignacio (1998), "Valoración bursátil de las empresas agroalimentarias", Investigación agraria. Producción 
y protección vegetales, 13 (3), Madrid, Instituto Nacional de Investigación y Tecnología Agraria y Alimentaria, pp. 319-344.

Calatrava Requena, Javier (2003), "Método de Precios Hedónicos: Posibilidades de aplicación en Valoración Ambiental", Ponencia presentada en el curso de doctorado 2002-2003, Evaluación Económica y Social de los Recursos Naturales, 1 de abril de 2003, Córdoba, Universidad de Córdoba.

Carrasco Gómez, Luis Antonio y Martel Montenegro, Alexander Melvin (2017), "Propuesta de nuevos métodos para estimar el valor comercial de inmuebles ubicados en la zona de "Mesa Redonda" (Cercado de Lima) como aporte al Reglamento Nacional de Tasaciones-2017”, tesis de maestría, Universidad San Martín de Porres, Lima.

CELSS (Congreso del Estado Libre y Soberano de Sinaloa) (2016), "Ley de Catastro del Estado de Sinaloa”, Tomo CVII, Culiacán, Congreso del Estado Libre y Soberano de Sinaloa, pp. 3-22.

Charnes, Abraham y Cooper, William Wager (1968), "Management Models and Industrial Applications of Linear Programming", Management Science, 4 (1), Catonsville, The Institute for Operations Research and the Management Sciences, pp. 38-91.

Diakoulaki, Danai; Mavrotas, George y Papayannakis, Lefteris (1995), "Determining objective weights in multiple criteria problems: The critic method", Computers and Operations Research, 22 (7), Nueva York, Elsevier, pp. 763-770, doi: doi.org/10.1016/03050548(94)00059-H

Escobar Urmenerta, María Teresa y Moreno-Jiménez, José María (1997), "Problemas de gran tamaño en el Proceso Analítico Jerárquico", Estudios de Economía Aplicada, núm. 8, Almería, Universidad de Almería, pp. 25-40.

García-Alcaraz, Jorge Luis y Noriega Morales, Salvador Anacleto (2008), "Evaluación multicriterio y multiatributos de tractores agrícolas: Un modelo y caso de estudio", Agricultura técnica en México, 34 (4), Texcoco, Instituto Nacional de Investigaciones Forestales, Agrícolas y Pecuarias, pp. 397-405. 
Garcia-Almirall, Pilar (2007), Introducción a la valoración inmobiliaria, Barcelona, Centre de Política de Sòl i Valoracions.

Hartwich, Frank (1998), "Weighting of Agricultural Research Results: Strength and Limitations of the Analytic Hierarchy Process (AHP). Research Evaluation and Weighting of Multiple Research Outputs", Documento de Trabajo, Stuttgart, Universität Hohenheim.

Humarán Nahed, Iván (2010). "Hacia una medida integrada del factor de localización en la valoración residencial: El caso de Mazatlán”, tesis de doctorado, Universitat Politècnica de Catalunya, Barcelona.

Inegi (Instituto Nacional de Estadística y Geografía) (2017), Anuario estadístico y geográfico de Sinaloa 2017, Aguascalientes, Inegi.

IVSC (International Valuation Standards Council) (2017), "International Valuation Standards: 2017”, Property Journal, Londres, IVSC.

Jahan, Ali; Mustapha, Faizal; Sapuan, S. M.; Ismail, Md Yusof y Bahraminasab, Marjan (2012), "A framework for weighting of criteria in ranking stage of material selection process", The International Journal of Advanced Manufacturing Technology, 58 (1-4), Nueva York, Springer, pp. 411-420, doi: doi.org/10.1007/s00170-0113366-7

Li, Zhiming; Fan, Zhengxi y Shen, Shiguang (2018), "Urban green space suitability evaluation based on the AHP-CV combined weight method: A case study of Fuping county, China”, Sustainability, 10 (8), Basel, MDPI, pp. 1-15, doi: doi.org/10.3390/su10082656

Linares Llamas, Pedro (1999), "Integración de criterios medioambientales en procesos de decisión: Una aproximación multicriterio a la planificación integrada de recursos eléctricos", tesis de doctorado, Universidad Politécnica de Madrid, Madrid.

Liu, Dinglin y Zhao, Xianglian (2013), "Method and application for dynamic comprehensive evaluation with subjective and objective information", PLoS ONE, 8 (12), San Francisco, Public Library of Science, pp. 1-5, doi: doi.org/10.1371/journal.pone.0083323

Llombart Bosch, María José (1996), "Catastro y equidad fiscal", Revista Hacienda Municipal, 16 (55), Ciudad de México, INDETEC, pp. 
16-24, <https://biblat.unam.mx/es/revista/revista-hacienda-municipal/articulo/catastro-y-equidad-fiscal>, 16 de marzo de 2018.

López-Ospina, Héctor Andrés y López Ospina, Rafael David (2010), "Modelos de optimización por metas para el cálculo de estimadores en regresión múltiple", Ciencia e Ingeniería Neogranadina, 20 (1), Bogotá, Editorial Neogranadina, pp. 133-157, doi: doi. org/10.18359/rcin.285

Madrid, Jorge Luis (2010), “Aplicación de métodos multicriterio, en la selección y ponderación, para la valuación de bienes inmuebles”, tesis de maestría, Instituto Tecnológico de la Construcción, Hermosillo, Sonora.

Martínez Blasco, Isabel (1998), "Modelos Econométricos Aplicados a la Valoración de Bienes Inmuebles Rústicos”, CT Catastro, núm. 134, Madrid, Ministerio de Hacienda, pp. 181-212.

Moreno-Jiménez, José María; Aguarón Joven, Juan; Cano Sevilla, Francisco y Escobar Urmeneta, María Teresa (1998), "Validez, robustez y estabilidad en decisión multicriterio. Análisis de sensibilidad en el proceso analítico jerárquico", Revista de la Real Academia de Ciencias Exactas, Físicas y Naturales, 92 (4), Madrid, Universidad Complutense de Madrid, pp. 387-397, <http://eprints.ucm. es/20720/1/Cano53.pdf>, 19 de marzo de 2019.

Moreno-Jiménez, José María (1996), "Metodología multicriterio en el plan nacional de regadíos", Documento de Trabajo, Zaragoza, Universidad de Zaragoza.

Mu, Enrique y Pereyra Rojas, Milagros (2017), "Understanding the Analytic Hierarchy Process", en Enrique Mu, Milagros Pereyra Rojas, Practical Decisión Making, Nueva York, Springer, pp. 7-22, doi: doi.org/10.1007/978-3-319-33861-3

Núñez Tabales, Julia Margarita; Ceular Villamandos, Nuria y Caridad Ocerín, José María (2008), "Metodología de precios hedónicos vs. redes neuronales artificiales como alternativas a la valoración de inmuebles. Un caso real", CT: Catastro, núm. 68, Madrid, Ministerio de Hacienda, pp. 27-42.

Olson, David (1988), "Opportunities and limitations of AHP in multiobjective programming", Mathematical and Computer Modelling, 
11 (C), Nueva York, Elsevier, pp. 206-209, doi: doi. org/10.1016/0895-7177(88)90481-5

PDDUCM (Plan Director de Desarrollo Urbano de la Ciudad de Mazatlán) (2013), "Plan Director de Desarrollo Urbano de la Ciudad de Mazatlán, Sinaloa”, Mazatlán, H. Ayuntamiento de Mazatlán.

Pellice, Rodolfo (2008), Valuación de Inmuebles, tomo 2, San Juan, Universidad Nacional de San Juan.

POES (Periódico Oficial del Estado de Sinaloa) (2016), "Ley de Catastro del Estado de Sinaloa", Culiacán, Sinaloa, 24 de junio, <http:// www.laipsinaloa.gob.mx/po_files/2016/junio/POE-24-06-2016077.pdf>, 15 de febrero de 2019.

Ridker, Ronald y Henning, John (1967), "The determinants of residential property values with special reference to air pollution", The Review of Economics and Statistics, 49 (2), Cambridge, MITPress, pp. 246-257, doi: doi.org/10.2307/1928231

Roca Cladera, Josep (1986), Manual de Valoraciones Inmobiliarias, Barcelona, Ariel.

SJF (Semanario Judicial de la Federación) (1976), "Impuestos, elementos esenciales de los. Deben estar consignados expresamente en la ley”, SJF, núm. 165, Ciudad de México, Suprema Corte de Justicia de la Nación, 15 de febrero de 2018.

SJF (Semanario Judicial de la Federación) (2000), "Impuestos. Principio de equidad tributaria previsto por el artículo 31, fracción IV, constitucional", SJF, núm. 35, Ciudad de México, Suprema Corte de Justicia de la Nación, 3 de marzo de 2018.

Torrado Fonseca, Mercedes y Reguant Alvarez, Mercedes (2016), "El Método Delphi", Revista d'Innovació i Recerca en Educació, 9 (1), Barcelona, Universitàt de Barcelona, pp. 87-102, doi: doi. org/10.1344/reire2016.9.1916.

Torres Asomoza, Luis Enrique (2006), "La justicia tributaria en los impuestos con fines extrafiscales", tesis de licenciatura, Universidad de las Américas Puebla, Puebla. 
Varela Ruiz, Margarita; Díaz Bravo, Laura y García Durán, Rocío (2012), "Descripción y usos del método Delphi en investigaciones del área de la salud", Investigación en Educación Médica, 1 (2), Ciudad de México, Elsevier, pp. 90-95.

Yalcin, Neşe y Ünlü, Ulaş (2017), "A multi-criteria performance analysis of initial public offering (IPO) firms using CRITIC and vikor methods", Technological and Economic Development of Economy, 24 (2), Vilnius, Taylor \& Francis, pp. 534-560, doi: doi.org/10. 3846/20294913.2016.1213201.

Recibido: 12 de enero de 2020. Reenviado: 6 de mayo de 2020. Aceptado: 15 de julio de 2020.

Luis Alfonso Colado Velázquez. Maestro en valuación inmobiliaria por la Universidad Autónoma de Durango, actualmente cursa el programa doctoral en Gestión y Valoración Urbana y Arquitectónica de la Universidad Politécnica de Cataluña, con adscripción en la Escuela de Ingeniería Mazatlán de la Universidad Autónoma de Sinaloa. Su línea de investigación es la valoración inmobiliaria.

Josep Roca Cladera. Doctor en arquitectura. Actualmente es catedrático de Arquitectura legal, Derecho urbanístico y Valoraciones del Departamento de Construcciones Arquitectónicas I y director del Centro de Política de Suelo y Valoraciones (CPSV) de la Universitat Politècnica de Catalunya, impulsor del Laboratorio de Modelización Virtual de la Ciudad, en el marco del CPSV y la Escuela T.S. Arquitectura y el Departamento de Expresión Gráfica. Sus líneas de investigación se enfocan en cambio climático, estudios metropolitanos, valoración de propiedad, planificación urbana y regional, ecología urbana, islas de calor urbano, clima urbano, efectos ambientales térmicos urbanos, asentamientos humanos y estudios urbanos. Entre sus últimas publicaciones se encuentran, como coautor: "Preferencias de la demanda sobre los materiales de construcción del inmueble residencial: caso de estudio con el Análisis Conjunto Adaptativo", ACE: Architecture, City and Environment, 15 (43), Barcelona, Universitat Politècnica de Catalunya, pp. 1-22 (2020); "Consecuencias hidrológicas del cambio climático en entornos urbanos", ponencia presentada en XIII CTV 2019 Proceedings: XIII International Conference on Virtual Cityand Territory: "Challenges and paradigms of the contemporarycity", 2-4, October, Barcelona, (2019) y "China’s population policies: past, present and future.", ACE: Architecture, City and Environ- 
ment, 12 (36), Barcelona, Universitat Politècnica de Catalunya, pp. 201218 (2018).

Iván Humarán Nahed. Doctor en gestión y valoración urbana y arquitectónica por la Universidad Politécnica de Cataluña; líder del cuerpo académico del Centro de Estudios de Valoraciones y Estudios Urbanos; profesor-investigador tiempo completo en la Universidad Autónoma de Sinaloa. Sus líneas de investigación se enfocan en valoraciones inmobiliarias. Entre sus últimas publicaciones se encuentran, como coautor: "Tecnología de drones, herramienta para el valuador inmobiliario", Revista de Investigación en Tecnologías de la Información: (RITI), 7 (13), Barcelona, Sistema Educativo de Investigación Científica e Innovación Tecnológica (SEICIT), Universitat Politècnica de Catalunya-Universidad Autónoma de Sinaloa pp. 117-125 (2019); "Formación y Distribución Espacial de los Valores Inmobiliarios: El Caso de Mazatlán”, Contexto, 11 (14), Nuevo León, Universidad Autónoma de Nuevo León, pp. 77-103 (2017) y "Hacia una media integrada del factor de localización en la valoración residencial: el caso de Mazatlán”, ACE: Architecture, City and Environment, 5 (13), Barcelona, Universitat Politècnica de Catalunya, pp. 185-218 (2010). 